\title{
Permanent pacemaker failure due to surgical emphysema
}

\author{
STEPHEN A SMITH, PETER L WEISSBERG, LIP-BUN TAN \\ From the University Department of Cardiovascular Medicine, East Birmingham Hospital, Birmingham
}

SUMmaRy A 76 year old man developed a left pneumothorax after implantation of a permanent endocardial pacemaker via the left subclavian vein. The resulting surgical emphysema caused temporary failure of the pacing system.

Permanent pacing systems that use a unipolar electrode require close contact between the pulse generator and surrounding tissues to complete the pacing circuit. Occasionally, electrode insertion via the subclavian vein is complicated by puncture of the lung apex causing a pneumothorax. We report a patient who developed a pneumothorax immediately after insertion of a permanent pacemaker.

\section{Case report}

A 76 year old man presented in August 1984 with a three week history of dizziness and dyspnoea on exertion. Physical examination showed mild congestive cardiac failure with a heart rate of 30 beats $/ \mathrm{min}$, and the electrocardiogram confirmed the presence of complete heart block. There was no previous history of cardiac or pulmonary disease. Temporary pacing with a bipolar electrode, positioned in the apex of the right ventricle via the right subclavian vein, resulted in rapid clinical improvement.

Two days later a permanent pacing system was implanted under local anaesthesia. A Siemens unipolar tined electrode was introduced into the right ventricular apex via the left subclavian vein. Some difficulty was experienced with the venous cannulation, and the left lung apex was punctured during the procedure. A satisfactory electrode position was, however, achieved, and the unit immediately functioned well with a pacing threshold of $0.3 \mathrm{~V}$ and an impedance of $528 \mathrm{Ohm}$. The pulse generator, a

Requests for reprints to Dr S A Smith, Department of Cardiology, East Birmingham Hospital, Bordesley Green East, Birmingham B9 5ST.
Biotronik Leptos MO1/2B, was positioned in a left pectoral subcutaneous pocket. The temporary electrode was left in situ.

Shortly after returning to the ward he became dyspnoeic with clinical signs of a left pneumothorax, which was confirmed by $x$ ray examination. An intercostal drain was inserted in the mid-axillary line and the lung expanded promptly. Three days later the permanent pacemaker suddenly failed with loss of the pacing artefacts on the electrocardiogram. A moderate amount of surgical emphysema had developed in the left anterior chest wall (Fig. 1), and crepitations were palpable around the pulse generator. Application of light pressure over the generator resulted in the immediate return of ventricular pacing, which ceased when the pressure was released (Fig. 2). Heart rate was maintained with the temporary pacemaker for a further four days, by which time the surgical emphysema had resolved and the permanent system resumed normal function. The intercostal drain and the temporary electrode were removed, and recovery was thereafter uneventful.

\section{Discussion}

Permanent pacemakers that use unipolar electrodes require good contact between the pulse generator and the pocket to complete the pacing circuit. Pacemaker failure in this case was caused by air collecting around the pulse generator, isolating its earthplate from the surrounding tissues. When pressure was applied over the generator this air was displaced, the circuit was completed, and pacing reinstituted. We are not aware 


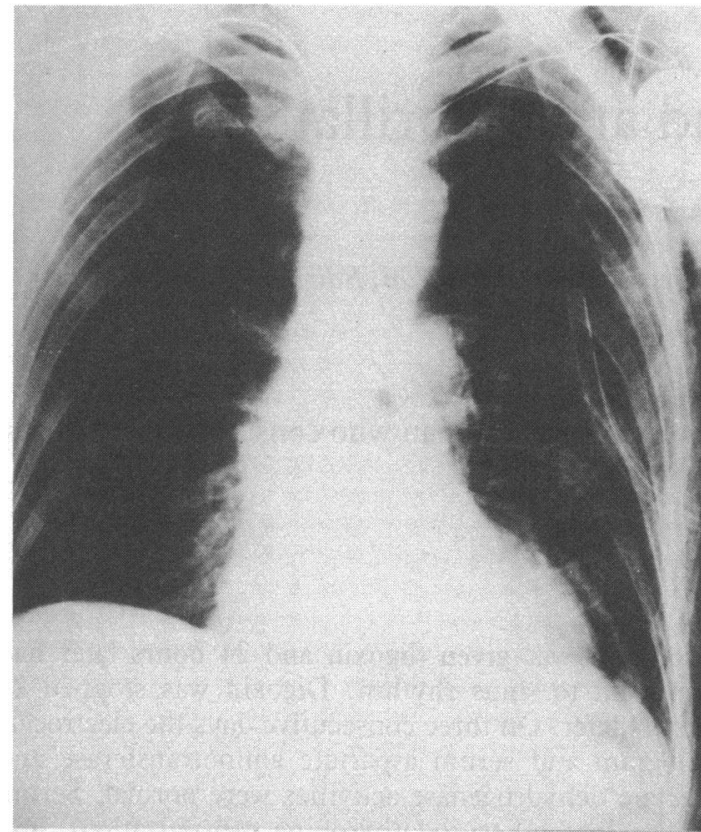

Fig. 1 -ray film showing the position of the chest drain and pulse generator and the surrounding surgical emphysema.

of any previous reports of permanent pacemaker failure attributed to this cause.

Even in the most experienced hands pneumothorax occurs in up to $5 \%$ of patients after subclavian cannulation, ${ }^{12}$ and so it is likely that this type of problem will occur again. We would recommend therefore that a temporary electrode be left in situ after permanent pacemaker insertion if the subclavian cannulation proves difficult and pulmonary damage is suspected.

Pacemaker failure in the early postoperative period is usually caused by electrode displacement, which requires further surgical intervention. If, however, permanent pacemaker failure with loss of pacing artefacts occurs in the presence of surgical emphysema then a conservative approach to management is indicated. Pacemaker function is likely to return as the emphysema resolves. In our patient air was able to enter the pulse generator pocket by tracking along the tissue plane from which the pocket had been formed. The risk of developing this complication may not be so great when healing has occurred and the generator is encapsulated in fibrous tissue but it cannot be entirely discounted. All permanent pacemaker dependent patients who develop surgical emphysema near the pulse generator at any time, and from any cause, should, therefore, be closely monitored for this type of pacemaker failure.

\section{References}

1 Bernard RW, Stahi WM. Subclavian vein catheterisation: a prospective study. 1 . Non-infectious complications. Ann Surg 1971; 173: 184-90.

2 Borja AR. Current status of infraclavicular subclavian vein catheterisation: a review of the English literature. Ann Thorac Surg 1972; 13: 615-24.

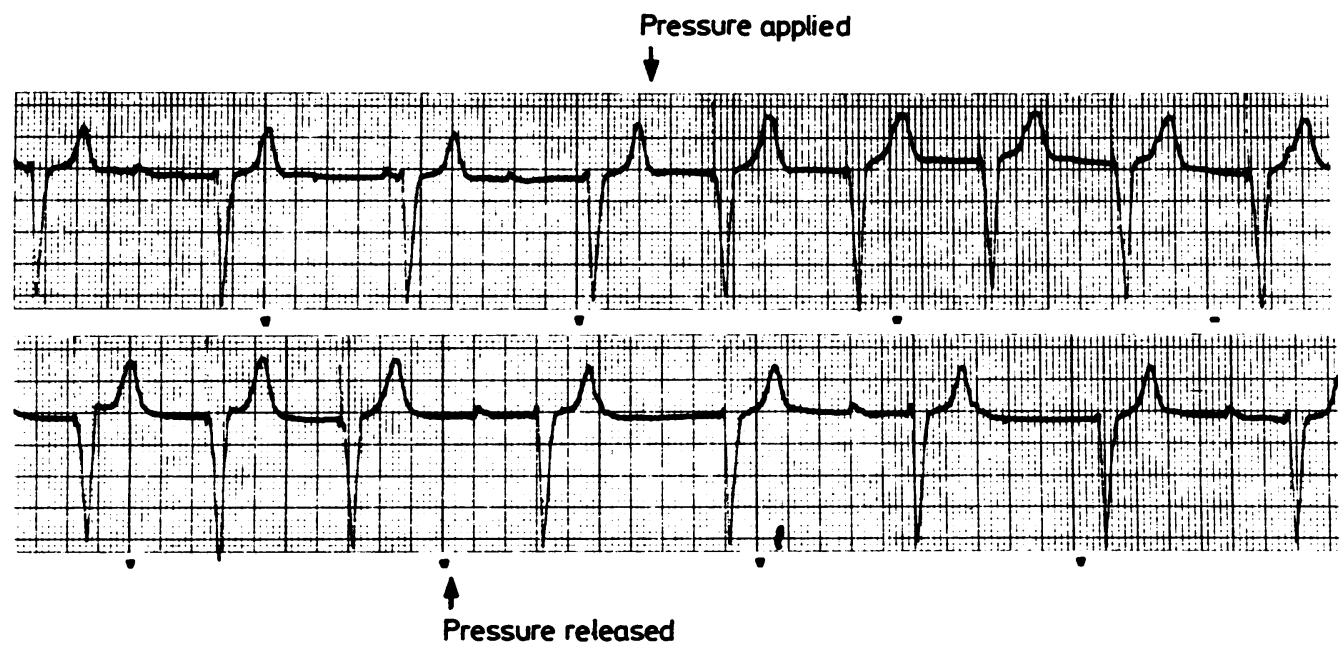

Fig. 2 Electrocardiogram showing the effect of pressure on the pulse generator. Before and after pressure is applied the rate is maintained at 50 beats /min by the temporary pacemaker with no visible pacing artefact from the permanent system. While pressure is applied the permanent box works normally at a rate of 70 beats/min. 